\title{
Putting Polyhedral Loop Transformations to Work
}

Cédric Bastoul — Albert Cohen — Sylvain Girbal — Saurabh Sharma — Olivier Temam

$\mathbf{N}^{\circ} 4902$

Juillet 2003

THÈME 1 



\title{
Putting Polyhedral Loop Transformations to Work
}

\author{
Cédric Bastoul $^{* \dagger}$, Albert Cohen* , Sylvain Girbal ${ }^{\ddagger * \S}$, Saurabh Sharma* \\ Olivier Temam ${ }^{\ddagger}$ \\ Thème 1 - Réseaux et systèmes \\ Projet A3
}

Rapport de recherche $n^{\circ} 4902$ - Juillet 2003 - 26 pages

\begin{abstract}
We seek to extend the scope and efficiency of iterative compilation techniques by searching not only for the most appropriate program transformation parameters but for the most appropriate transformations themselves, or even for compositions of transformations. For that purpose, we need to find a generic way to express program transformations and compositions of transformations. In this article, we introduce a framework for the polyhedral representation of a wide range of program transformations in a unified and generic way. We also show that it is possible to generate efficient code after the application of polyhedral program transformations. Finally, we demonstrate an implementation of the program transformation framework and the code generation techniques in the Open64/ORC compiler.
\end{abstract}

Key-words: Automatic parallelization, optimization, loop nests, polyhedral transformations, code generation

\footnotetext{
* A3 group, INRIA Rocquencourt

$\dagger$ PRiSM, University of Versailles

$\ddagger$ LRI, Paris South University

$\S$ LIST, CEA Saclay
} 


\section{Mise en Pratique des Transformation Polyédriques de Boucles}

Résumé : Nous cherchons à étendre l'applicabilité et l'efficacité des techniques de compilation itératives, en cherchant non seulement les paramètres de transformation les plus appropriés, mais aussi les meilleures transformations elles mêmes, voire les compositions de transformations. Pour cela, nous avons besoin d'une écriture générique pour exprimer des transformations et des compositions de transformations. Dans cet article, nous introduisons un cadre général pour la représentation polyédrique d'une large classe de transformations, de manière unifiée et générique. Nous montrons également qu'il est possible de générer du code efficace après application des transformations de programmes. Enfin, nous décrivons une implémentation de ce cadre général et de ses techniques de génération de code dans le compilateur Open64/ORC.

Mots-clés : Parallélisation automatique, optimisation, nids de boucles, transformations polyédriques, génération de code 


\section{Introduction}

Optimizing and parallelizing compilers face a tough challenge. Due to their impact on productivity and portability, programmers of high-performance applications want compilers to automatically produce quality code on a wide range of architectures. Simultaneously, Moore's law indirectly urges the architects to build complex architectures with deeper pipelines and (non uniform) memory hierarchies, wider generalpurpose and embedded cores with clustered units and speculative structures. Aggressive program transformations targeting parallelism and memory hierarchies have matured since the first manual techniques, but the static cost models implemented in compilers have a hard time coping with rapidly increasing architecture complexity. Recent research works on iterative and feedback-directed optimizations [1] suggest that practical approaches based on dynamic, i.e., execution-time, information can better harness complex architectures.

Current approaches to iterative optimizations usually choose a rather small set of program transformations, e.g., cache tiling and array padding, and focus on finding the best possible transformation parameters, e.g., tile size and padding size [2] using parameter search space techniques. However, a recent comparative study of model-based vs. empirical optimizations [3] stresses that many motivations for iterative, feedback-directed or dynamic optimizations are irrelevant when the proper transformations are not available.

Within our research group, we want to extend the scope and efficiency of iterative compilation techniques by making the program transformation itself one of the parameters, so that searching the parameter space means both finding the appropriate program transformation and the appropriate program transformation parameters. Moreover, we want to search for composition of program transformations and not only single program transformations. For that purpose, we need to have a generic method for expressing program transformations and composition of those, and this method should be amenable to parameter search space techniques.

This article introduces a unified framework for implementing program transformations, and composition of program transformations in a generic way. This framework relies on a polyhedral representation of loops and loop transformations. By separating the iteration domains from the statement and iteration schedules, and by enabling per-statement transformations, this representation avoids many of the limitations of iteration-based program transformations, widens the set of possible program transformations and provides a parameterized expression of program trans-

formations. Although a few invariants constrain the search space, we beleive this

$\mathrm{RR} \mathrm{n}^{\circ} 4902$ 
generic expression of program transformations is appropriate for systematic search space techniques.

The corresponding search techniques are out of the scope of this work and will be investigated in a follow-up article. This work presents the principles of our unified framework and the first part of its implementation. Also, since polyhedral transformation techniques can better accommodate complex control structures than traditional loop-based transformations, we start with an empirical study of control structures within a set of benchmarks. The four key aspects of our research work are: (1) empirically evaluating the scope of polyhedral program transformations, (2) defining a practical transformation environment based on a polyhedral representation, (3) showing that it is possible to generate efficient code from a polyhedral transformation, (4) implementing the polyhedral representation and code generation technique in a real compiler, Open64/ORC [4].

Eventually, our framework operates at an abstract semantical level to hide the details of the control structures, rather than on a syntax tree. This eases the design and implementation of complex program transformations, such as combined schedule and data-layout reordering [5]. We also define useful extensions of existing techniques, such as per-statement and versatile transformations that make few assumptions about control structures and loop bounds. Consequently, while our framework is initially geared toward iterative optimization techniques, it can also facilitate the implementation of statically driven program transformations in a traditional optimizing compiler.

The paper is organized as follows. We present the empirical analysis of static control structures in Section 2 and discuss their significance in typical benchmarks. The unified transformation model is described in Section 3, followed by the composition of polyhedral transformations in Section 4. Section 5 presents the code generation techniques used after polyhedral transformations. Finally, the implementation of these different techniques in Open64/ORC is described in Section 6.

\section{Static Control Parts}

Let us start with some related works. Since we did not directly contribute to the driving of optimizations and parallelization techniques, we will not compare with the vast literature in the field of model-based and empirical optimization.

Well-known loop restructuring compilers proposed unified models and intermediate representations for loop transformations, but none of them addressed the general composition and parameterization problem of polyhedral techniques. ParaScope [6] 
is both a dependence-based framework and an interactive source-to-source compiler for Fortran; it implements classical loop transformations. SUIF [7] was designed as an intermediate representation and framework for automatic loop restructuring; it quickly became a standard platform for implementing virtually any optimization prototype, with multiple front-ends, machine-dependent back-ends and variants. Polaris [8] is an automatic parallelizing compiler for Fortran; it features a rich sequence of analyzes and loop transformations applicable to real benchmarks. These three projects are based on a syntax-tree representation, ad-hoc dependence models and implement polynomial algorithms. PIPS [9] is probably the most complete loop restructuring compiler, implementing polyhedral analyses and transformations (including affine schedulng) and interprocedural analyses (array regions, alias). PIPS uses an expressive intermediate representation, a syntax-tree with polyhedral annotations.

Within the Omega project [10], the Petit dependence analyzer and loop restructuring tool [11] is closer to our work: it provides a unified polyhedral framework for iteration reordering only, and it shares our emphasis on per-statement transformations. It is intended as a research tool for compute-intensive kernels.

Two codesign tools share a lot of motivations and technology with our semiautomatic optimization project. MMAlpha [12] is a domain-specific single assignment language for systolic array computations, a polyhedral transformation framework, and a high-level circuit synthesis tool. The interactive and semi-automatic approach to polyhedral transformations were introduced by MMAlpha. The PICO project [13] is a more pragmatic approach to codesign, restricting the application domain to loop nests with uniform dependences and aiming at the selection and coordination of existing functional units to generate an application-specific VLIW processor. Both tools target compute-intensive kernels only.

\subsection{Decomposition into Static Control Parts}

In the following, loops are normalized and split in two categories:

- loops from 0 to some bound expression with an integer stride, called do loops;

- other kinds of loops, referred to as while loops.

In our implementation, early phases of the Open64 compiler perform most of this normalization, along with closed form substitution of induction variables. Notice some Fortran and $\mathrm{C}$ while loops may be normalized to do loops when bound and stride can be discovered statically.

RR $\mathrm{n}^{\circ} 4902$ 
The following definition is a slight extension of static control nests [14]. Within a function body, a static control part $(\mathrm{SCoP})$ is a maximal set of consecutive statements without while loops, where loop bounds and conditionals may only depend on invariants within this set of statements. These invariants include symbolic constants, formal function parameters and surrounding loop counters: they are called the global parameters of the SCoP, as well as any invariant appearing in some array subscript within the $\mathrm{SCoP}$. A static control part is called rich when it holds at least one non-empty loop; rich SCoPs are the natural candidates for polyhedral loop transformations. An example is shown in Figure 1. We will only consider rich SCoPs int he following.

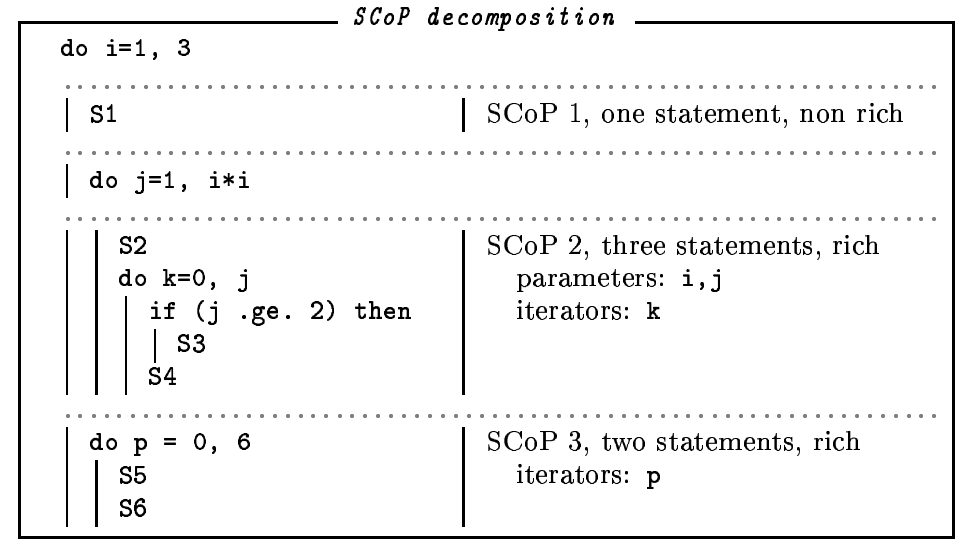

Figure 1: Example of decomposition into static control parts

As such, a SCoP may hold arbitrary memory accesses and function calls; a SCoP is thus larger than a static control loop nest [14]. Interprocedural alias and array region analysis would be useful for precise dependence analysis. Nevertheless, our semi-automatic framework copes with crude dependence information in authorizing the expert user to override static analysis when applying transformations. Moreover, extensions to "sparsely-irregular" codes (with a few unpredictable conditionals and while loops) are possible [15, 16], although a "fully" polyhedral representation is not possible anymore. Unifying hybrid static-dynamic optimization [17] with the polyhedral model would also be interesting.

\subsection{Automatic Discovery of SCoPs}


SCoP extraction is greatly simplified when implemented within a modern compiler infrastructure such as Open64/ORC. Previous phases include function inlining, constant propagation, loop normalization, integer comparison normalization, dead-code and goto elimination, and induction variable substitution, along with languagespecific preprocessing: pointer arithmetic is replaced with indexed arrays, pointer analysis information is available (but not yet used in our tool), etc.

The algorithm for SCoP extraction proceeds through the following steps:

Gather useful information: traverse the syntax tree of a given function (after inlining) and store do loop counters, bounds and strides, conditional predicates, array references, and parameters involved in these expressions.

\section{Recognize affine information:}

- among do loops, select the static control ones by checking the bound expressions and the invariance of the loop counter and parameters within the loop body;

- select the static control conditionals whose predicate is an affine expression of parameters and loop counters;

- restrict previous static control loops to those enclosing static control loops and static control conditionals;

- select the array references whose subscript is an affine expression of parameters and loop counters.

Build static control parts: traverse the syntax tree, starting with the creation of a new SCoP. Let $N$ be the first operational or control node in the loop body:

- if $N$ is a static control loop, add this loop and its enclosed statements to the $\mathrm{SCoP}$;

- if $N$ is a static control conditional, add this conditional with its branches to the $\mathrm{SCoP}$;

- if $N$ is not a conditional or loop node, add it to the SCoP;

- otherwise, close the current SCoP and create a new one;

- drop the SCoP if it does not contain at least one loop.

Then, set $N$ to the next node and continue the traversal.

Identify the global parameters: for each SCoP, iterate over the loop bounds, conditional expressions and array subscripts to gather the global parameters.

$\mathrm{RR} \mathrm{n}^{\circ} 4902$ 
This algorithm outputs a list of SCoPs associated with any function in the syntax tree. Our implementation in Open64 is discussed in Section 6.

\subsection{Significance Within Real Applications}

Thanks to an implementation of the previous algorithm into Open64, we studied the applicability of our polyhedral framework to several benchmarks.

Figure 2 summarizes the results for the SpecFP 2000 and PerfectClub benchmarks handled by our tool (single-file programs only, at the time being). All codes are written in Fortran77, except for art and quake in C, and lucas in Fortran90. The first column shows the number of functions, outlining that code modularity does not seem to impact the applicability of polyhedral transformations (inlining was not applied in these experiments). The next two columns count the number of SCoPs with at least one global parameter and enclosing at least one conditional, respectively; the first one advocates for parametric analysis and transformation techniques; the second one shows the need for techniques that handle static-control conditionals. The next two columns in the "Statements" section shows that SCoPs hold a large majority of statements which reinforces the coverage of static control parts, and also illustrates the computationally intensive nature of the benchmarks (many statements are enclosed in loops). The last two columns in the "Array References" section are very promising for dependence analysis: most subscripts are affine except for lucas and $\mathrm{mg} 3 \mathrm{~d}$; moreover, the rate is over $99 \%$ in 7 benchmarks, but approximate array dependence analyses will be required for an good coverage of the 5 other benchmarks.

In accordance with earlier results using Polaris [18], the coverage of regular loop nests is strongly influenced by the quality of the constant propagation, loop normalization and induction variable detection.

Our tool also gathers detailed statistics about the number of parameters and statements per SCoP, and about statement depth (within a SCoP, not counting nonstatic enclosing loops). Figure 3 shows that almost all SCoPs are smaller than 100 statements, with a few exceptions, and that loop depth is rarely greater than 3 . Moreover, deep loops also tend to be very small, except for applu, adm and mg3d which contain depth-3 loop nests with tenths of statements. This means that most polyhedral analysis and transformations will succeed and require only a reasonable amount of time and resources. It also gives an estimate of the scalability required for worst-case exponential algorithms, like the code generation phase to convert the polyhedral representation back to source code. 


\begin{tabular}{|c|c|c|c|c|c|c|c|c|}
\hline & \multirow[b]{2}{*}{ Functions } & \multicolumn{3}{|c|}{ SCoPs } & \multicolumn{2}{|c|}{ Statements } & \multicolumn{2}{|c|}{ Array References } \\
\hline & & All & Parametric & Affine ifs & All & in SCoPs & All & Affine \\
\hline applu & 16 & 19 & $\overline{15}$ & $\overline{1}$ & $\overline{757}$ & 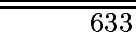 & 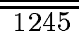 & $100 \%$ \\
\hline apsi & 97 & 80 & 80 & 25 & 2192 & 1839 & 977 & $78 \%$ \\
\hline art & 26 & 28 & 27 & 4 & 499 & 343 & 52 & $100 \%$ \\
\hline lucas & 4 & 4 & 4 & 2 & 2070 & 2050 & 411 & $40 \%$ \\
\hline mgrid & 12 & 12 & 12 & 2 & 369 & 369 & 176 & $99 \%$ \\
\hline quake & 27 & 20 & 14 & 4 & 639 & 489 & 218 & $100 \%$ \\
\hline swim & 6 & 6 & 6 & 1 & 123 & 123 & 192 & $100 \%$ \\
\hline$\overline{\mathrm{adm}}$ & $\overline{997}$ & $\overline{80}$ & $\overline{\overline{80}}$ & $\overline{25}$ & 2260 & $\overline{1899}$ & $\overline{147}$ & $\overline{\overline{95 \%}}$ \\
\hline dyfesm & 78 & 75 & 70 & 3 & 1497 & 1280 & 507 & $99 \%$ \\
\hline $\mathrm{mdg}$ & 16 & 17 & 17 & 5 & 530 & 464 & 355 & $84 \%$ \\
\hline mg3d & 28 & 39 & 39 & 6 & 1442 & 1242 & 1274 & $19 \%$ \\
\hline qcd & 35 & 30 & 23 & 6 & 819 & 641 & 943 & $100 \%$ \\
\hline
\end{tabular}

Figure 2: Coverage of static control parts in high-performance applications
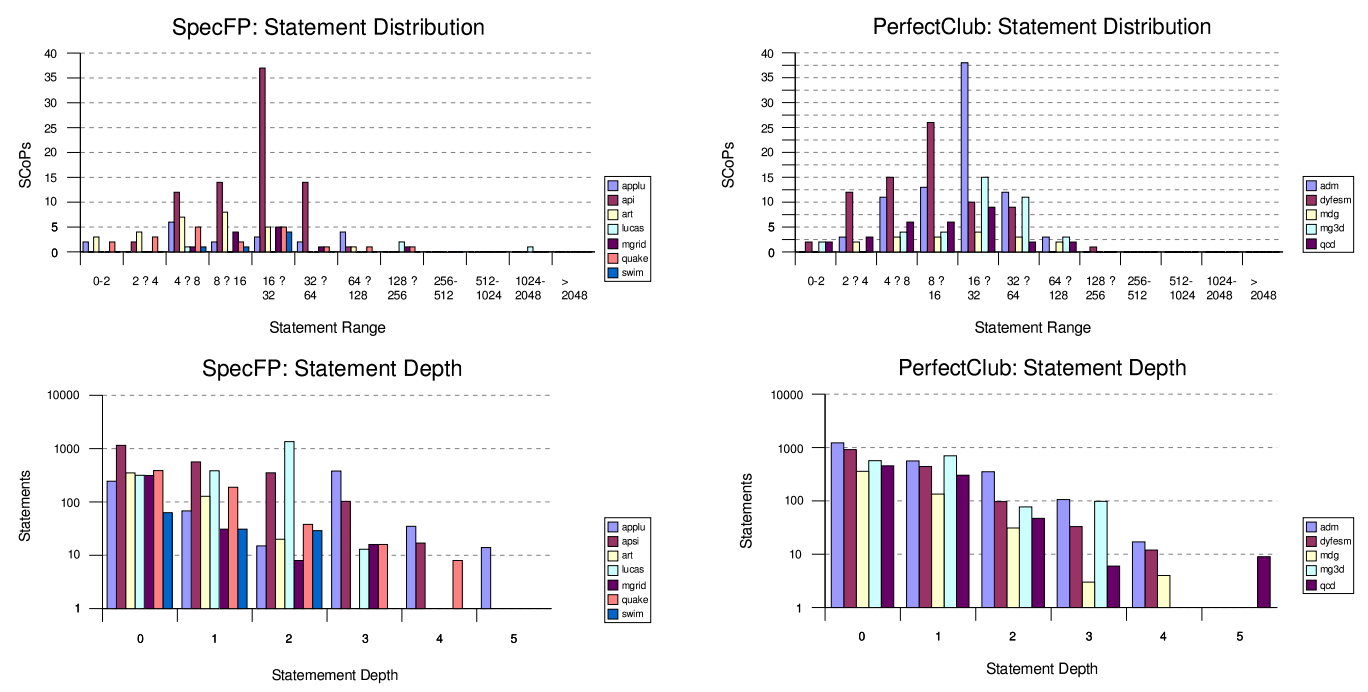

Figure 3: Distribution of statement depths and SCoP size

\section{Unified Polyhedral Representation}

In this section, we define the principles of polyhedral program transformations. The term polyhedron will be used in a broad sense to denote a convex set of points in a lattice (also called $\mathbb{Z}$-polyhedron or lattice-polyhedron), i.e., a set of points in a $\mathbb{Z}$ vector space bounded by affine inequalities [19].

$\mathrm{RR} \mathrm{n}^{\circ} 4902$ 
Let us now introduce the representation of a SCoP and its elementary transformations. A static control part within the syntax tree is a pair $\left(\mathcal{S}, \mathbf{i}_{\mathrm{gp}}\right)$, where $\mathcal{S}$ is the set of consecutive statements - in their polyhedral representation - and $\mathbf{i}_{\mathrm{gp}}$ is the vector of global parameters of the SCoP. Vector $\mathbf{i}_{\mathrm{gp}}$ is constant for the SCoP but statically unknown; yet its value is known at runtime, when entering the SCoP. $d_{\mathrm{gp}}=\operatorname{dim}\left(\mathbf{i}_{\mathrm{gp}}\right)$ denotes the number of global parameters.

We will use a few specific linear algebra notations:

- matrices are always denoted by capital letters, vectors and functions in vector spaces are not;

- $\operatorname{pfx}(v, n)$ returns a length- $n$ prefix of $v$, i.e., the vector built from the $n$ first components of $v$;

- $u \sqsubseteq w$ is equivalent to $u$ being a prefix of $v$;

- $1_{k}$ denotes the $k$-th unit vector in a reference base $\left(1_{1}, \ldots, 1_{d}\right)$ of a $d$-dimensional space, i.e., $(0, \ldots, 0,1,0, \ldots, 0)$; likewise, $1_{i, j}$ denotes the matrix filled with zeros but element $(i, j)$ set to 1 .

A SCoP may also be decorated with static properties related with the polyhedral representation, such as array dependences [14] or regions [20], but this work does not address static analysis.

\subsection{Domains, Schedules and Access Functions}

The depth $d^{S}$ of a statement $S$ is the number of nested loops enclosing $S$ in the SCoP. A statement $S \in \mathcal{S}$ is a quadruple $\left(\mathcal{D}^{S}, \mathcal{L}^{S}, \mathcal{R}^{S}, \theta^{S}\right)$, where $\mathcal{D}^{S}$ is the $d^{S}$ dimensional iteration domain of $S, \mathcal{L}^{S}$ and $\mathcal{R}^{S}$ are sets of polyhedral representations of array references, and $\theta^{S}$ is the affine schedule of $S$, defining the sequential execution ordering of iterations of $S$. To represent arbitrary lattice polyhedra, each statement is provided with a number $d_{\mathrm{lp}}^{S}$ of local parameters to implement integer division and modulo operations via affine projection: e.g., the set of even values of $i$ is described by means of a local parameter $p$ - existentially quantified - and equation $i=2 p$. Let us describe these concepts in more detail and give some examples.

- $\mathcal{D}^{S}$ is a convex polyhedron defined by matrix $\Lambda^{S} \in \mathcal{M}_{n, d^{S}+d_{\mathrm{lp}}^{S}+d_{\mathrm{gp}}+1}(\mathbb{Z})$ such that

$$
\mathbf{i} \in \mathcal{D}^{S} \Longleftrightarrow \exists \mathbf{i}_{\mathrm{lp}}, \Lambda^{S}\left(\begin{array}{c}
\mathbf{i} \\
\mathbf{i}_{\mathrm{lp}} \\
\mathbf{i}_{\mathrm{gp}} \\
1
\end{array}\right) \geq 0 .
$$


Notice the last matrix column is always multiplied by the constant 1 ; it corresponds to the homogeneous coordinate encoding of affine inequalities into linear form. The number $n$ of constraints in $\Lambda^{S}$ is not limited. Program statements guarded by non-convex conditionals - such as $0 \leq i \leq 3 \vee i \geq 8$ - are split into separate statements with convex domains in the polyhedral representation.

\section{Example.}

The Figure 4 show an example that illustrates these definitions.

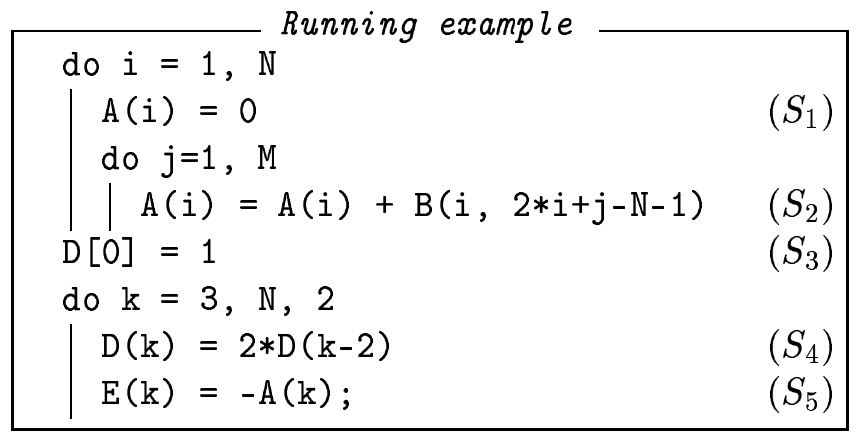

Figure 4: Running example

The domains of the five statements are:

$\mathcal{D}^{S_{1}}=\{i \mid 1 \leq i \leq N\}$

$\mathcal{D}^{S_{2}}=\{(i, j) \mid 1 \leq i \leq N, 1 \leq j \leq M\}$

$\mathcal{D}^{S_{3}}=\{()\}$ (the zero-dimensional vector)

$\mathcal{D}^{S_{4}}=\mathcal{D}^{S_{5}}=\{k \mid 3 \leq k \leq N \wedge \exists p, k=3+2 p\}$

So, the $\Lambda$-matrix for those statements are:

$$
\begin{aligned}
& \Lambda^{S_{1}}=\left[\begin{array}{cccc}
1 & 0 & 0 & -1 \\
-1 & 1 & 0 & 0
\end{array}\right] \\
& \Lambda^{S_{2}}=\left[\begin{array}{ccccc}
1 & 0 & 0 & 0 & -1 \\
-1 & 0 & 1 & 0 & 0 \\
0 & 1 & 0 & 0 & -1 \\
0 & -1 & 0 & 1 & 0
\end{array}\right] \\
& \begin{array}{l|l}
\text { with } & \begin{array}{l}
\mathbf{i}=(i) \\
\mathbf{i}_{\mathrm{lp}}=() \\
\mathbf{i}_{\mathrm{gp}}=(N, M)
\end{array}
\end{array} \\
& \Lambda^{S_{3}}=\text { ] } \\
& \Lambda^{S_{4}}=\Lambda^{S_{5}}=\left[\begin{array}{ccccc}
1 & 0 & 0 & 0 & -3 \\
-1 & 0 & 1 & 0 & 0 \\
1 & -2 & 0 & 0 & -3 \\
-1 & 2 & 0 & 0 & 3
\end{array}\right] \text { with } \quad \begin{array}{l}
\mathbf{i}=(k) \\
\mathbf{i}_{\mathrm{lp}}=(p) \\
\mathbf{i}_{\mathrm{gp}}=(N, M)
\end{array}
\end{aligned}
$$


- $\mathcal{L}^{S}$ and $\mathcal{R}^{S}$ describe array references written by $S$ (left-hand side) or read by $S$ (right-hand side), respectively; it is a set of pairs (A, $f$ ) where A is an array variable and $f$ is the access function mapping iterations in $\mathcal{D}^{S}$ to locations in A. The access function $f$ is defined by a matrix $\mathrm{F} \in \mathcal{M}_{\operatorname{dim}(\mathrm{A}), d^{S}+d_{\mathrm{lp}}^{S}+d_{\mathrm{gp}}+1}(\mathbb{Z})$ such that

$$
f(\mathbf{i})=\exists \mathbf{i}_{\mathrm{lp}}, \mathrm{F}\left(\begin{array}{c}
\mathbf{i} \\
\mathbf{i}_{\mathrm{lp}} \\
\mathbf{i}_{\mathrm{gp}} \\
1
\end{array}\right) .
$$

\section{Example.}

Consider statement $S_{2}$ of previous example:

$$
\mathcal{L}^{S_{2}}=\{(\mathrm{A},(i))\} \quad \mathcal{R}^{S_{2}}=\left\{(\mathrm{A},(i)),\left(\mathrm{B},\left(\begin{array}{c}
i \\
2 * i+j-N-1
\end{array}\right)\right)\right\}
$$

and they are stored as:

$$
\begin{aligned}
\mathcal{L}^{S_{2}} & =\left\{\left(\mathrm{A},\left[\begin{array}{lllll}
1 & 0 & 0 & 0 & 0
\end{array}\right]\right)\right\} \\
\mathcal{R}^{S_{2}} & =\left\{\left(\mathrm{A},\left[\begin{array}{lllll}
1 & 0 & 0 & 0 & 0
\end{array}\right]\right),\left(\mathrm{B},\left[\begin{array}{ccccc}
1 & 0 & 0 & 0 & 0 \\
2 & 1 & -1 & 0 & -1
\end{array}\right]\right)\right\}
\end{aligned}
$$

with

$$
\mid \begin{aligned}
& \mathbf{i}=(i, j) \\
& \mathbf{i}_{\mathrm{lp}}=() \\
& \mathbf{i}_{\mathrm{gp}}=(N, M)
\end{aligned}
$$

- $\theta^{S}$ is the affine schedule of $S$; it maps iterations in $\mathcal{D}^{S}$ to time-stamps (i.e., logical execution dates) in $2 d^{S}+1$-dimensional time [21]. Multidimensional time-stamps are compared through the lexicographic ordering over vectors, denoted by «: iteration $\mathbf{i}$ of $S$ is executed before iteration $\mathbf{i}^{\prime}$ of $S^{\prime}$ if and only if $\theta^{S}(\mathbf{i}) \ll \theta^{S}\left(\mathbf{i}^{\prime}\right)$.

To facilitate code generation and to schedule independently iterations and statements, we need $2 d^{S}+1$ time dimensions instead of $d^{S}$ (the minimum for an affine sequential schedule). This encoding was first proposed by Feautrier [21] and used extensively by Kelly and Pugh [11]: dimension $2 k$ encodes the relative ordering of statements at depth $k$ and dimension $2 k-1$ encodes the ordering of iterations in loops at depth $k$. 
Eventually, $\theta^{S}$ is defined by a matrix $\Theta^{S} \in \mathcal{M}_{2 d^{S}+1, d^{S}+d_{\mathrm{gp}}+1}(\mathbb{Z})$ such that

$$
\theta^{S}(\mathbf{i})=\Theta^{S}\left(\begin{array}{c}
\mathbf{i} \\
\mathbf{i}_{\mathrm{gp}} \\
1
\end{array}\right)
$$

Notice $\Theta^{S}$ does not involve local parameters, since lattice polyhedra do not increase the expressivity of sequential schedules.

\section{Example.}

The $\Theta$-matrices from the previous example are:

$$
\begin{aligned}
& \theta^{S_{1}}(\mathbf{i})=\left(\begin{array}{l}
0 \\
i \\
0
\end{array}\right) \text { stored as } \Theta^{S_{1}}=\left[\begin{array}{llll}
0 & 0 & 0 & 0 \\
1 & 0 & 0 & 0 \\
0 & 0 & 0 & 0
\end{array}\right] \text { with } \begin{array}{l}
\mathbf{i}=(i) \\
\mathbf{i}_{\mathrm{lp}}=() \\
\mathbf{i}_{\mathrm{gp}}=(N, M)
\end{array} \\
& \theta^{S_{2}}(\mathbf{i})=\left(\begin{array}{c}
0 \\
i \\
1 \\
j \\
0
\end{array}\right) \text { stored as } \Theta^{S_{2}}=\left[\begin{array}{lllll}
0 & 0 & 0 & 0 & 0 \\
1 & 0 & 0 & 0 & 0 \\
0 & 0 & 0 & 0 & 1 \\
0 & 1 & 0 & 0 & 0 \\
0 & 0 & 0 & 0 & 0
\end{array}\right] \text { with } \begin{array}{l}
\mathbf{i}=(i, j) \\
\mathbf{i}_{\mathrm{pp}}=() \\
\mathbf{i}_{\mathrm{gp}}=(N, M)
\end{array} \\
& \theta^{S_{3}}(\mathbf{i})=\left(\begin{array}{l}
1
\end{array}\right) \text { stored as } \Theta^{S_{3}}=\left[\begin{array}{lll}
0 & 0 & 1
\end{array}\right] \text { with } \begin{array}{l}
\mathbf{i}=() \\
\mathbf{i}_{\mathrm{lp}}=() \\
\mathbf{i}_{\mathrm{gp}}=(N, M)
\end{array} \\
& \theta^{S_{4}}=\left(\begin{array}{l}
2 \\
k \\
0
\end{array}\right) \text { stored as } \Theta^{S_{4}}=\left[\begin{array}{llll}
0 & 0 & 0 & 2 \\
1 & 0 & 0 & 0 \\
0 & 0 & 0 & 0
\end{array}\right] \text { with } \begin{array}{l}
\mathbf{i}=(k) \\
\mathbf{i}_{\mathrm{lp}}=() \\
\mathbf{i}_{\mathrm{gp}}=(N, M)
\end{array} \\
& \theta^{S_{5}}=\left(\begin{array}{c}
2 \\
k \\
1
\end{array}\right) \text { stored as } \Theta^{S_{5}}=\left[\begin{array}{llll}
0 & 0 & 0 & 2 \\
1 & 0 & 0 & 0 \\
0 & 0 & 0 & 1
\end{array}\right] \text { with } \mid \begin{array}{l}
\mathbf{i}=(k) \\
\mathbf{i}_{\mathrm{l}_{\mathrm{p}}}=() \\
\mathbf{i}_{\mathrm{gp}}=(N, M)
\end{array}
\end{aligned}
$$

\subsection{Invariants}

We will now present an important contribution of this work: our representation makes a clear separation between the semantically meaningful transformations expressible on the polyhedral representation from the semantically safe transformations satisfying the statically checkable properties. The goal is of course to relax as many constraints on the source code as possible to widen the range of meaningful transformations without relying on the accuracy of a static analyzer. For example, many loop transformations are hampered by the lack of information about the bounds or the limitation to whole-block operations: in most cases, a polyhedral representation

$\mathrm{RR} \mathrm{n}^{\circ} 4902$ 
hides these difficulties in separating the domains from the schedules and by authorizing transparent statementwise operations. To reach this goal, and also to achieve a high degree of transformation compositionality, the polyhedral representation enforces a few invariants on the domains and schedules.

There is only one domain invariant. To avoid integer overflows (in the transformation and code generation phases), the coefficients in a row of $\Lambda^{S}$ must be relatively prime:

$$
\forall 1 \leq i \leq d^{S}, \operatorname{gcd}\left(\Lambda_{i, 1}, \ldots, \Lambda_{i, d_{\mathrm{gp}}+1}\right)=1 .
$$

This restriction has no effect on the expressible domains.

The first schedule invariant is that the schedule matrix must fit into a decomposition more amenable to expert-driven transformation and code generation. It separates the square iteration reordering matrix $\mathrm{A}^{S} \in \mathcal{M}_{d^{S}, d^{S}}(\mathbb{Z})$ operating on iteration vectors, from the parameterized matrix $\Gamma^{S} \in \mathcal{M}_{d^{S}, d_{\mathrm{gp}+1}}(\mathbb{Z})$ and from the statement-scattering vector $\beta^{S} \in \mathbb{N}^{d^{S}+1}$ :

$$
\Theta^{S}=\left[\begin{array}{ccccccc}
0 & \cdots & 0 & 0 & \cdots & 0 & \beta_{0}^{S} \\
\mathrm{~A}_{1,1}^{S} & \cdots & \mathrm{A}_{1, d^{S}}^{S} & \Gamma_{1,1}^{S} & \cdots & \Gamma_{1, d_{\mathrm{gp}}}^{S} & \Gamma_{1, d_{\mathrm{gp}}+1}^{S} \\
0 & \cdots & 0 & 0 & \cdots & 0 & \beta_{1}^{S} \\
\mathrm{~A}_{2,1}^{S} & \cdots & \mathrm{A}_{2, d^{S}}^{S} & \Gamma_{2,1}^{S} & \cdots & \Gamma_{2, d_{\mathrm{gp}}}^{S} & \Gamma_{2, d_{\mathrm{gp}}+1}^{S} \\
\vdots & \ddots & \vdots & 0 & \ddots & 0 & \vdots \\
\mathrm{A}_{d^{S}, 1}^{S} & \cdots & \mathrm{A}_{d^{S}, d^{S}}^{S} & \Gamma_{d^{S}, 1}^{S} & \cdots & \Gamma_{d^{S}, d_{\mathrm{gp}}}^{S} & \Gamma_{d^{S}, d_{\mathrm{gp}}+1}^{S} \\
0 & \cdots & 0 & 0 & \cdots & 0 & \beta_{d^{S}}^{S}
\end{array}\right] .
$$

Dimensions associated with statement scattering may not depend on loop counters or parameters, hence the zeroes in "even dimensions". We will show in Section 3.4 how most primitive transformations operate on $\mathrm{A}^{S}, \beta^{S}$ or $\Gamma^{S}$ separately. Notice $\beta$ subscripts range from 0 to $d^{S}$.

Back to the running example, matrix $\Theta^{S_{2}}$ splits into

$\Theta^{S_{2}}=\left[\begin{array}{lllll}0 & 0 & 0 & 0 & 0 \\ 1 & 0 & 0 & 0 & 0 \\ 0 & 0 & 0 & 0 & 1 \\ 0 & 1 & 0 & 0 & 0 \\ 0 & 0 & 0 & 0 & 0\end{array}\right] \longrightarrow \mathrm{A}^{S_{2}}=\left[\begin{array}{ll}1 & 0 \\ 0 & 1\end{array}\right] \wedge \Gamma^{S_{2}}=\left[\begin{array}{lll}0 & 0 & 0 \\ 0 & 0 & 0\end{array}\right] \wedge \beta^{S_{2}}=\left[\begin{array}{l}0 \\ 1 \\ 0\end{array}\right]$

The second schedule invariant is the sequentiality one: two distinct statement iterations may not have the same time-stamp:

$$
S \neq S^{\prime} \vee \mathbf{i} \neq \mathbf{i}^{\prime} \Rightarrow \theta^{S}(\mathbf{i}) \neq \theta^{S^{\prime}}\left(\mathbf{i}^{\prime}\right) \text {. }
$$


Whether the iterations belong to the domain of $S$ and $S^{\prime}$ does not matter in (3): we wish to be able to transform iteration domains without bothering with the sequentiality of the schedule. Because this invariant is hard to enforce directly, we introduce two additional invariants with no impact on schedule expressivity and stronger than $(3)$ :

$$
\begin{gathered}
\left|\operatorname{det}\left(\mathrm{A}^{S}\right)\right|=1 \text {, i.e., } \mathrm{A}^{S} \text { is unimodular, } \\
S \neq S^{\prime} \Rightarrow \beta^{S} \neq \beta^{S^{\prime}} .
\end{gathered}
$$

Finally, we add a density invariant to avoid integer overflow and ease schedule comparison. The "odd dimensions" of the image of $\Theta^{S}$ form a $d^{S}$-dimensional sub-space of the multidimensional time, since $\mathrm{A}^{S}$ is unimodular, but an additional requirement is needed to enforce that "even dimensions" satisfy some form of dense encoding:

$$
\beta_{k}^{S}>0 \Rightarrow \exists S^{\prime} \in \mathcal{S}, \operatorname{pfx}\left(\beta^{S}, k\right)=\operatorname{pfx}\left(\beta^{S^{\prime}}, k\right) \wedge \beta_{k}^{S^{\prime}}=\beta_{k}^{S}-1,
$$

i.e., for a given prefix, the next dimension of the statement-scattering vectors span an interval of non-negative integers.

\subsection{Constructors}

We define some elementary functions on SCoPs, called constructors. Many matrix operations consist in adding or removing a row or column. Given a vector $v$ and matrix $\mathrm{M}$ with $\operatorname{dim}(v)$ columns and at least $i$ rows, $\operatorname{AddRow}(\mathrm{M}, i, v)$ inserts a new row at position $i$ in $\mathrm{M}$ and fills it with the value of vector $v$, whereas $\operatorname{Rem} \operatorname{Row}(\mathrm{M}, i)$ does the opposite transformation. Analogous constructors exist for columns, AddCol(M, $j, v)$ inserts a new column at position $j$ in $\mathrm{M}$ and fills it with vector $v$, whereas $\operatorname{Rem} \operatorname{Col}(\mathrm{M}, j)$ undoes the insertion. AddRow and RemRow are extended to operate on vectors.

Displacement of a statement $S$ is also a common operation. It only impacts the statement-scattering vector $\beta^{S^{\prime}}$ of some statements $S^{\prime}$ sharing some common property with $S$. Indeed, forward or backward movement of $S$ at depth $\ell$ triggers the same movement on every subsequent statement $S^{\prime}$ at depth $\ell$ such that $\operatorname{pfx}\left(\beta^{S^{\prime}}, \ell\right)=$

$\operatorname{pfx}\left(\beta^{S}, \ell\right)$. Although rather intuitive, the following definition with prefixed blocks of statements is rather technical. Consider a $\operatorname{SCoP} \mathcal{S}$, a statement-scattering prefix $P$ defining the depth at which statements should be displaced, a statement-scattering prefix $Q$ - prefixed by $P$ - making the initial time-stamp of statements to be displaced, and a displacement distance $o$; $o$ is the value to be added/subtracted to the component at depth $\operatorname{dim}(P)$ of any statement-scattering vector $\beta^{S}$ prefixed by $P$

$\mathrm{RR} \mathrm{n}^{\circ} 4902$ 
and following $Q$. The displacement constructor Move leave all statements unchanged except those satisfying the following conditions:

$$
\operatorname{Move}(P, Q, o): \quad \forall S \in \mathcal{S}, P \sqsubseteq \beta^{S} \wedge\left(Q \ll \beta^{S} \vee Q \sqsubseteq \beta^{S}\right): \beta_{\operatorname{dim}(P)}^{S} \leftarrow \beta_{\operatorname{dim}(P)}^{S}+o
$$

Notice these constructors make no assumption about the representation invariants and may violate them.

\subsection{Primitives}

From the earlier constructors, we will now define transformation primitives that enforce the invariants and serve as building blocks for higher level, semantically sound transformations. Most primitives correspond to simple polyhedral operations, but their formal definition is rather technical and will be described more extensively in a further paper. Figure 5 lists the main primitives affecting the polyhedral representation of a statement. ${ }^{1} \mathrm{U}$ denotes a unimodular matrix; $\mathrm{M}$ implements the parameterized shift (or translation) of the affine schedule of a statement; $\ell$ denotes the depth of a statement insertion, iteration domain extension or restriction; and $c$ is a vector implementing an additional domain constraint.

The last two primitives - fusion and split (or distribution) - show the benefit of designing loop transformations at the abstract semantical level of polyhedra. First of all, loop bounds are not an issue since the code generator will handle any overlapping of iteration domains. Next, these primitives do not directly operate on loops, but consider prefixes $P$ of statement-scattering vectors. As a result, they may virtually be composed with any possible transformation. For the split primitive, vector $(P, o)$ prefixes all statements concerned by the split; and parameter $b$ indicates the position where statement delaying should occur. For the fusion primitive, vector $(P, o+1)$ prefixes all statements that should be interleaved with statements prefixed by $(P, o)$. Eventually, notice that fusion followed by split (with the appropriate value of $b$ ) leaves the SCoP unchanged.

This table is not complete: privatization, array contraction and copy-propagation require primitives operating on the access functions of individual array references. This will be the purpose of another paper.

Finally, embedding static analysis in the primitives raises no fundamental problem; but updating the analyzes' meta-information across transformations is still under investigation.

\footnotetext{
${ }^{1}$ As a cosmetic improvement, many of these primitives can be extended to work on blocks of statements sharing a common statement-scattering prefix (along the lines of the fusion and splitting primitives).
} 


\begin{tabular}{|c|c|c|}
\hline Syntax \& Name & Prerequisites & Effect \\
\hline $\begin{array}{l}\operatorname{LEFTU}(S, \mathrm{U}) \\
\text { Unimodular } \\
\end{array}$ & $\begin{array}{l}S \in \mathcal{S} \wedge U \in \mathcal{M}_{d^{S}, d^{S}}(\mathbb{Z}) \\
\wedge|\operatorname{det}(\mathrm{U})|=1\end{array}$ & $\overline{\mathrm{A}^{S}} \leftarrow \mathrm{U} \cdot \mathrm{A}^{S}$ \\
\hline $\begin{array}{l}\text { RIGHTU }(S, \mathrm{U}) \\
\text { Unimodular }\end{array}$ & $\begin{array}{l}S \in \mathcal{S} \wedge U \in \mathcal{M}_{d^{S}, d^{S}}(\mathbb{Z}) \\
\wedge|\operatorname{det}(\mathrm{U})|=1\end{array}$ & $\mathrm{~A}^{S} \leftarrow \mathrm{A}^{S} \cdot \mathrm{U}$ \\
\hline $\begin{array}{l}\text { SHIFT }(S, M) \\
\text { Shift }\end{array}$ & $S \in \mathcal{S} \wedge \mathrm{M} \in \mathcal{M}_{d^{S}, d_{\mathrm{gp}+1}(\mathbb{Z})}$ & $\Gamma^{S} \leftarrow \Gamma^{S}+\mathrm{M}$ \\
\hline $\begin{array}{l}\operatorname{INSERT}(S, \ell) \\
\text { Insertion }\end{array}$ & $\begin{array}{l}\ell \leq d^{S} \wedge \beta_{\ell+1}^{S}=\cdots=\beta_{d S}^{S}=0 \\
\wedge\left(\exists S^{\prime} \in \mathcal{S}, \operatorname{pfx}\left(\beta^{S}, \ell+1\right) \sqsubseteq \beta^{S^{\prime}}\right. \\
\left.\quad \vee\left(\operatorname{pfx}\left(\beta^{S}, \ell\right), \beta_{\ell}^{S}-1\right) \sqsubseteq \beta^{S^{\prime}}\right)\end{array}$ & $\begin{array}{l}P=\operatorname{pfx}\left(\beta^{S}, \ell\right) \\
\mathcal{S} \leftarrow \operatorname{Move}\left(P,\left(P, \beta_{\ell}^{S}\right), 1\right) \cup S\end{array}$ \\
\hline $\begin{array}{l}\text { Delete }(S) \\
\text { Deletion }\end{array}$ & $S \in \mathcal{S}$ & $\begin{array}{l}P=\operatorname{pfx}\left(\beta^{S}, d^{S}\right) \\
\mathcal{S} \leftarrow \operatorname{Move}\left(P,\left(P, \beta_{d S}^{S}\right),-1\right) \backslash S\end{array}$ \\
\hline $\begin{array}{l}\operatorname{ExTEND}(S, \ell) \\
\text { Extension }\end{array}$ & $S \in \mathcal{S}$ & $\begin{array}{l}d^{S} \leftarrow d^{S}+1 ; \Lambda^{S} \leftarrow \operatorname{AddCol}\left(\Lambda^{S}, \ell, 0\right) \\
\mathrm{A}^{S} \leftarrow \operatorname{AddRow}\left(\operatorname{Add} \operatorname{Col}\left(\mathrm{A}^{S}, \ell, 0\right), \ell, 1_{\ell}\right) \\
\beta^{S} \leftarrow \operatorname{AddRow}\left(\beta^{S}, \ell, 0\right) ; \Gamma^{S} \leftarrow \operatorname{AddRow}\left(\Gamma^{S}, \ell, 0\right) \\
\forall(\mathrm{A}, \mathrm{F}) \in \mathcal{L}^{S} \cup \mathcal{R}^{S}, \mathrm{~F} \leftarrow \operatorname{AddRow}(\mathrm{F}, \ell, 0)\end{array}$ \\
\hline $\begin{array}{l}\text { RESTRICT }(S, \ell) \\
\text { Restriction }\end{array}$ & $S \in \mathcal{S}$ & $\begin{array}{l}d^{S} \leftarrow d^{S}-1 ; \Lambda^{S} \leftarrow \operatorname{Rem} \operatorname{Rol}\left(\Lambda^{S}, \ell\right) \\
\mathrm{A}^{S} \leftarrow \operatorname{RemRow}\left(\operatorname{Rem} \operatorname{Rol}\left(\mathrm{A}^{S}, \ell\right), \ell\right) ; \\
\beta^{S} \leftarrow \operatorname{RemRow}\left(\beta^{S}, \ell\right) ; \Gamma^{S} \leftarrow \operatorname{RemRow}\left(\Gamma^{S}, \ell\right) \\
\forall(\mathrm{A}, \mathrm{F}) \in \mathcal{L}^{S} \cup \mathcal{R}^{S}, \mathrm{~F} \leftarrow \operatorname{RemRow}(\mathrm{F}, \ell)\end{array}$ \\
\hline $\begin{array}{l}\text { CutDomain }(S, c) \\
\text { Cut Domain }\end{array}$ & $\begin{array}{l}S \in \mathcal{S} \\
\wedge \operatorname{dim}(c)=d^{S}+d_{1 \mathrm{p}}^{S}+d_{\mathrm{gp}}+1\end{array}$ & $\begin{array}{l}\Lambda^{S} \leftarrow \operatorname{AddRow}\left(\Lambda^{S}, 0,\right. \\
\left.\qquad / \operatorname{gcd}\left(c_{1}, \ldots, c_{d^{S}+d_{\mathrm{lp}}^{S}+d_{\mathrm{gp}}+1}\right)\right)\end{array}$ \\
\hline $\begin{array}{l}\text { ADDLP }(S) \\
\text { Add Local } \\
\text { Parameter } \\
\end{array}$ & $S \in \mathcal{S}$ & $\begin{array}{l}d_{\mathrm{lp}}^{S} \leftarrow d_{\mathrm{lp}}^{S}+1 \\
\Lambda^{S} \leftarrow \operatorname{AddCol}\left(\Lambda^{S}, d^{S}+1,0\right) ; \\
\forall(\mathrm{A}, \mathrm{F}) \in \mathcal{L}^{S} \cup \mathcal{R}^{S}, \mathrm{~F} \leftarrow \operatorname{Add} \operatorname{Col}\left(\mathrm{F}, d^{S}+1,0\right)\end{array}$ \\
\hline $\begin{array}{l}\text { FuSE }(P, o) \\
\text { Fusion }\end{array}$ & & $\begin{array}{l}b=\max \left\{\beta_{\operatorname{dim}(P)+1}^{S} \mid(P, o) \sqsubseteq \beta^{S}\right\}+1 ; \\
\operatorname{Move}((P, o+1),(P, o+1), b) ; \operatorname{Move}(P,(P, o+1),-1)\end{array}$ \\
\hline $\begin{array}{l}\operatorname{SPLIT}(P, o, b) \\
\text { Split }\end{array}$ & & $\operatorname{Move}(P,(P, o, b), 1) ; \operatorname{Move}((P, o+1),(P, o+1),-b)$ \\
\hline
\end{tabular}

Figure 5: Main transformation primitives

\section{Transformation Composition}

We will illustrate the composition of primitives on a typical example: two-dimensional tiling. To define such a composed transformation, we first build the strip-mining and interchange transformations from the primitives, as shown in Figure 6.

Interchange $(S, o)$ swaps the roles of $\mathbf{i}_{o}$ and $\mathbf{i}_{o+1}$ in the schedule of $S$; it is a perstatement extension of the classical interchange of $\mathbf{i}_{o}$ and $\mathbf{i}_{o+1} \cdot \operatorname{StripMine}(S, o, k)$ - where $k$ is a known integer — prepends a new iterator to virtually $k$-times unroll the schedule and iteration domain of $S$ at depth $o$. Finally, $\operatorname{Tile}(S, o, k)$ tiles the loops at depth $o$ and $o+1$ with $k \times k$ blocks.

This tiling transformation is a first step towards a higher-level combined transformation, integrating strip-mining and interchange with privatization, array copy

$\mathrm{RR} \mathrm{n}^{\circ} 4902$ 


\begin{tabular}{|c|c|c|c|}
\hline Syntax \& Name & Prerequisites & Effect & Comments \\
\hline $\begin{array}{l}\text { INTERCHANGE }(S, o) \\
\text { Loop Interchange }\end{array}$ & $\begin{array}{l}S \in \mathcal{S} \\
\wedge o<d^{S}\end{array}$ & $\begin{array}{l}U=I_{d S}-1_{o, o}-1_{o+1, o+1}+1_{o, o+1}+1_{o+1, o} \\
S \leftarrow \operatorname{RighT}(S, U)\end{array}$ & swap rows $o$ and $o+1$ \\
\hline $\begin{array}{l}\text { STRIPMine }(S, o, k) \\
\text { Strip Mining }\end{array}$ & $\begin{array}{l}S \in \mathcal{S} \\
\wedge o \leq d^{S} \\
\wedge k>0\end{array}$ & $\begin{array}{l}S \leftarrow \operatorname{Extend}(S, o) ; \\
S \leftarrow \operatorname{AddLP}(S) \\
p=d^{S}+1 ; \\
u=d^{S}+d_{\mathrm{lp}}^{S}+d_{\mathrm{gp}}+1 ; \\
S \leftarrow \operatorname{CutDomain}\left(S, 1_{o+1}-1_{o}\right) \\
S \leftarrow \operatorname{CutDomain}\left(S, 1_{o}-1_{o+1}+(k-1) \times 1_{u}\right) \\
S \leftarrow \operatorname{CutDomain}\left(S, 1_{o}-1_{p}\right) \\
S \leftarrow \operatorname{CutDomain}\left(S, 1_{p}-1_{o}\right) ;\end{array}$ & $\begin{array}{l}\text { local parameter column } \\
\text { constant column } \\
\left(\mathbf{i}_{o} \leq \mathbf{i}_{o+1}\right) \\
\left(\mathbf{i}_{o+1} \leq \mathbf{i}_{o}+k-1\right) \\
(k \times p \leq i i) \\
(i i \leq k \times p)\end{array}$ \\
\hline $\begin{array}{l}\text { TILE }(S, o, k) \\
\text { Tiling }\end{array}$ & $\begin{array}{l}S \in \mathcal{S} \\
\wedge o<d^{S} \\
\wedge k>0\end{array}$ & $\begin{array}{l}S \leftarrow \text { StripMine }(S, o, k) \\
S \leftarrow \text { StripMine }(S, o+2, k) \\
S \leftarrow \operatorname{Interchange}(S, o+1)\end{array}$ & \\
\hline
\end{tabular}

Figure 6: Composition of transformation primitives

propagation and hoisting for dependence removal. The only remaining parameters would be the statements and loops of interest and the tile size.

\section{Code Generation}

After polyhedral transformations, code generation is the last step to the final program. It is often ignored in spite of its impact on the target code quality. In particular, we must ensure that a bad control management does not spoil performance, for instance by producing redundant guards or complex loop bounds.

Ancourt and Irigoin [22] proposed the first solution, based on the Fourier-Motzkin pair-wise elimination [19]. The scope of their method was very restrictive, since it could be applied to only one polyhedron, with unimodular transformation (scheduling) matrices. The basic idea was to apply the transformation function as a change of base of the loop indices, then for each new dimension, to project the polyhedron on the axis and thus find the corresponding loop bounds. The main drawback of this method was the large amount of redundant control. Most further works on code generation tried to extend this first technique, in order to deal with non-unit strides $[23,24]$ or with a non-invertible transformation matrix [25]. A few alternatives to the Fourier-Motzkin were discussed, but without addressing the challenging problem of scanning more than one polyhedron with the same code.

This problem was first solved by generating a naive perfectly nested code and then by (partially) eliminating redundant guards [10]. Another way was to generate the code for each polyhedron separately, and then to merge them [25, 26]. This solution generates a lot of redundant control, even if there were no redundancies in 
the separated code. Quilleré et al. proposed to recursively generate a set of loop nests scanning several unions of polyhedra by separating them into subsets of disjoint polyhedra and generating the corresponding loop nests from the outermost to the innermost levels [27]. This later approach provides at present the best solutions since it guarantees that there is no redundant control. However, it suffers from some limitations, e.g. high complexity, code generation with unit strides only, and an arbitrary partial order on the polyhedra. Some improvements are presented in the next section.

This section presents the code generation problem, its resolution with a modern polyhedral-scanning technique, and its implementation.

\subsection{The Code Generation Problem}

In the polyhedral model, code generation amounts to a polyhedron scanning problem. We consider this problem as a two-part specification for each statement:

- A convex polyhedron, defined by a set of parameterized inequalities in the $n$ dimensional space:

$$
\mathcal{D}^{S}=\left\{\mathbf{i} \in \mathbb{Z}^{d^{S}} \mid \exists \mathbf{i}_{\mathrm{lp}}, \Lambda_{\mathrm{i}}^{S} \mathbf{i}+\Lambda_{\mathrm{lp}}^{S} \mathbf{i}_{\mathrm{lp}}+\Lambda_{\mathrm{gp}}^{S} \mathbf{i}_{\mathrm{gp}}+\lambda^{S} \geq 0\right\},
$$

this is the same definition as in Section 3.1 but $\Lambda$ is split according to the four parts of the homogeneous coordinate vector.

- A scheduling function, an affine function specifying a scanning order for the integral points belonging to the union of iteration domains:

$$
\theta^{S}(\mathbf{i})=\Theta_{\mathrm{i}}^{S} \mathbf{i}+\Theta_{\mathrm{gp}}^{S} \mathbf{i}_{\mathrm{gp}}+\beta^{S},
$$

again, $\Theta^{S}$ is split into three parts according to the homogeneous coordinate vector. Depending on the context, the scanning function may have several interpretations: to distribute the iterations across different processors, to order them in time, or both (by composition), etc.

The problem boils down to finding a set of nested loops visiting each integral point, following the scanning order. The generated code quality can be assessed by using two valuations: the most important is the amount of duplicated control in the final code; second, the code size, since a large code may pollute the instruction cache. We choose the recent Quilleré et al. method [27] with some additional improvements, which guarantee a code generation without any duplicated control. The outline of the modified algorithm is presented in Section 5.2 and some useful optimization are discussed in Section 5.3.

$\mathrm{RR} \mathrm{n}^{\circ} 4902$ 


\subsection{Outline of the Code Generation Algorithm}

Our code generation process is divided in two main steps. First, we take the scheduling functions into account by modifying each polyhedron's lexicographic order. Next, we use an improved Quilleré et al. algorithm to perform the actual code generation.

When no schedule is specified, the scanning order is the plain lexicographic order. Applying a new scanning order to a polyhedron amounts to adding new dimensions in leading positions. Thus, from each polyhedron $\mathcal{D}^{S}$ and scheduling function $\theta^{S}$, we build another polyhedron with the desired lexicographic order:

$\mathcal{T}^{S}=\left\{\left(\frac{\mathbf{t}}{\mathbf{i}}\right) \mid \exists \mathbf{i}_{\mathrm{lp}},\left[\begin{array}{c|c}I d & -\Theta_{\mathrm{i}}^{S} \\ \hline 0 & \Lambda_{\mathrm{i}}^{S}\end{array}\right]\left(\frac{\mathbf{t}}{\mathbf{i}}\right)+\left[\frac{0}{\Lambda_{\mathrm{lp}}^{S}}\right] \mathbf{i}_{\mathrm{lp}}+\left[\frac{-\Theta_{\mathrm{gp}}^{S}}{\Lambda_{\mathrm{gp}}^{S}}\right] \mathbf{i}_{\mathrm{gp}}+\left(\frac{-\beta^{S}}{\lambda^{S}}\right) \frac{=}{\geq} 0\right\}$.

By definition, $(\mathbf{t}, \mathbf{i}) \in \mathcal{T}^{S}$ if and only if $\mathbf{t}=\theta^{S}(\mathbf{i})$.

The main part of the algorithm is a recursive generation of the scanning code, maintaining a list of polyhedra from the outermost to the innermost loops. At each step, it:

1. intersects each polyhedron of the list with the context of the current loop (to restrict the scanning code to this loop);

2. projects the resulting polyhedra onto the outermost dimensions, then separate the projections into disjoint polyhedra;

3. sorts the resulting polyhedra such that a polyhedron is before another one if its scanning code has to precede the other to respect the lexicographic order;

4. merges successive polyhedra having at least another loop level to generate a new list and recursively generate the loops that scan this list;

5. computes the strides that the current dimension imposes to the outer dimensions.

This algorithm is slightly different from the one presented by Quilleré et al. in [27]; our two main contributions are the following: the support for non-unit strides (Step 5) and the exploitation of degrees of freedom (i.e., when some operations do not have a schedule) to produce a more effective code (Step 4).

Let us describe this algorithm with a non-trivial example. We propose to scan the two polyhedral domains presented in Figure 7(a). Both statements have iteration vector $(i, j)$, local parameter vector $(k)$ and global parameter vector $(n)$. We first 
compute the intersections with the context (i.e., at this point, the constraints on the global parameters, supposed to be $n \geq 6$ ). We project the polyhedra onto the first dimension, $i$, then we separate them into disjoint polyhedra. This means that we compute the domains where there are points to scan for $\mathcal{T}^{S_{1}}$ alone, both $\mathcal{T}^{S_{1}}$ and $\mathcal{T}^{S_{2}}$, and $\mathcal{T}^{S_{2}}$ alone (as shown in Figure 7(b), this last domain is empty). Here, we notice there is a local parameter implying a non-unit stride; we can determine this stride and update the lower bound. We finally generate the scanning code for this first dimension. We now recurse on the next dimension, repeating the process for each polyhedron list (in this example, there are now two lists: one inside each generated outer loop). We intersect each polyhedra with the new context, now the outer loop iteration domains; then we project the resulting polyhedra on the outer dimensions, and finally we separate these projections into disjoint polyhedra This last processing is trivial for the second list but yields two domains for the first list, as shown in Figure 7(c). Eventually, we generate the code associated with the new dimension, and since this is the last one, the scanning code is fully generated.

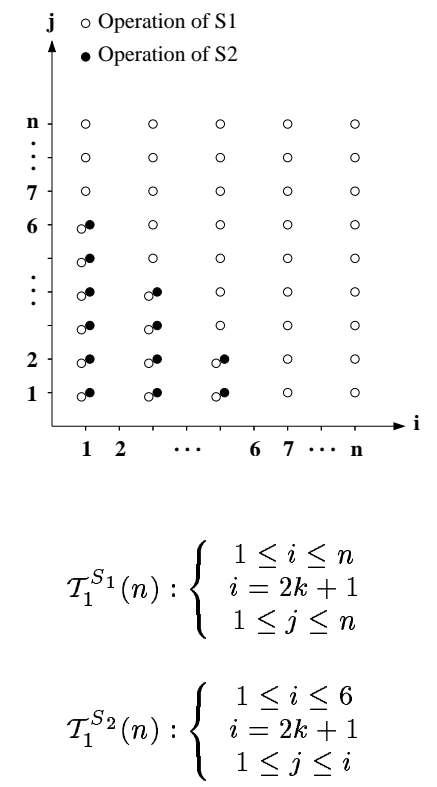

(a) Initial domains to scan
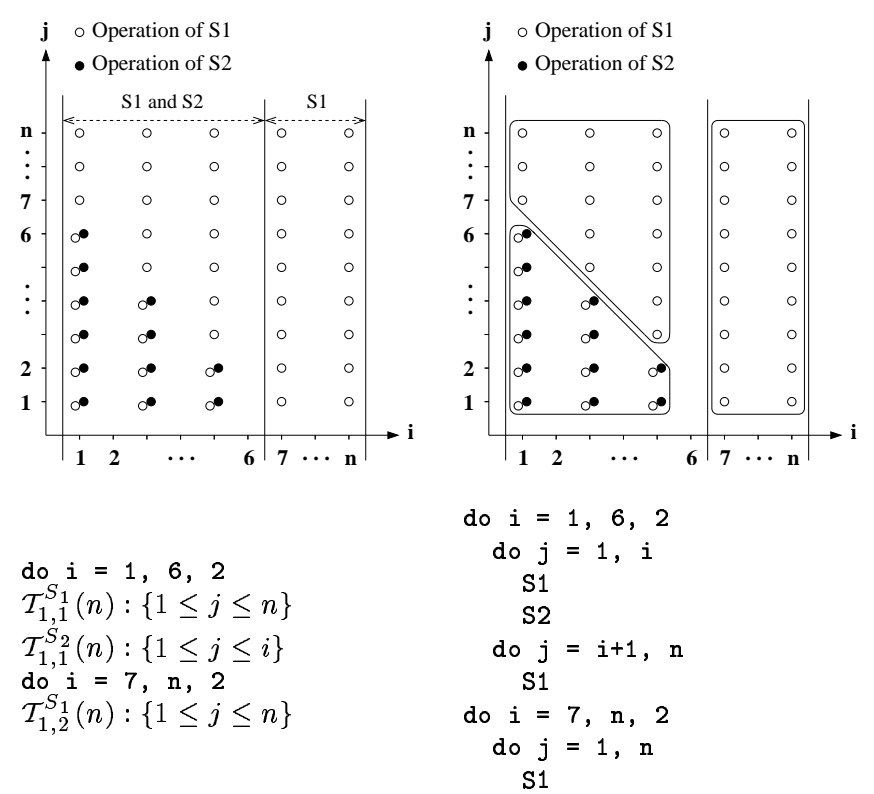

(b) Projection and separation on (c) Recursion on next dimensions

Figure 7: Step by step code generation example 


\subsection{Complexity Issues}

The main computing kernel in the code generation process is the separation into disjoint polyhedra. Given a list of $n$ polyhedra, we have to compute $\mathcal{T}^{S_{1}}-\mathcal{T}^{S_{2}}\left(S_{1}\right.$ alone), $\mathcal{T}^{S_{1}} \cap \mathcal{T}^{S_{2}}$ (both $S_{1}$ and $S_{2}$ ) and $\mathcal{T}^{S_{2}}-\mathcal{T}^{S_{1}}$ ( $S_{2}$ alone), then do the same for each resulting polyhedra with $S_{3}$, and so on. Thus, the worst-case complexity is $\mathcal{O}\left(3^{n}\right)$ polyhedral operations (exponential themselves). In addition, the memory usage is very high since we have to allocate memory for each separated domain. For both issues, we propose a partial solution.

- We use pattern matching to reduce the number of polyhedral computations: at a given depth, the domains are often the same (this is a property of the input codes), or disjoint (this is a property of the statement-scattering vectors of the scheduling matrices). We thus check these properties before any polyhedral operation and significantly improve performance.

- To avoid a memory allocation explosion, when we detect a high memory consumption, we continue the code generation process for the remaining recursions with a more naive algorithm, leading to a less efficient code but using far less memory.

Our implementation of this algorithm is called CLooG (Chunky Loop Generator) and was originally designed for a locality-improvement algorithm and software (Chunky) [5]. CLooG could regenerate code for all 12 benchmarks in Figure 2. It produced optimal control for all but two SCoPs in apsi and adm (the same loop nest, the two benchmarks being quasi-identical). These two SCoPs have less than 50 statements, but 16 parameters; since the current version of WRaP-IT does not analyse the linear relations vetween variables, the variability of parameter interactions leads to an exponential growth of the generated code. Notice the code generation for the large SCoP with more than 1700 statements in lucas took 22 minutes and $1 G B$ of memory on a Itanium $800 \mathrm{MHz}$ processor. Complexity improvements and studies of the generated code quality are under investigation.

\section{WRaP-IT: an Open64 Plug-In for Polyhedral Trans- formations}

Our main goal is to streamline the extraction of static control parts and the code generation, to ease the integration of polyhedral techniques into optimizing and parallelizing compilers. This interface tool is built on Open64/ORC. It converts the 
WHIRL - the compiler's hierarchical intermediate representation - to an augmented polyhedral representation, maintaining a correspondence between matrices in $\mathrm{SCoP}$ descriptions with the symbol table and syntax tree. This representation is called the WRaP: WHIRL Represented as Polyhedra. It is the basis for any polyhedral analysis or transformation. Then, the second part of the tool is a modified version of CLooG, to regenerate a WHIRL syntax tree from the WRaP. The whole Interface Tool is called WRaP-IT. Since its input and output are binary encoding of the WHIRL (Open64 .B or .N file), WRaP-IT may be used in a normal compilation flow as well as in a source-to-source framework. This versatile behaviour is best understood on the following example:

1. run Open64/ORC and suspend the compilation flow after loop normalization, induction variable substitution, and scalar optimizations;

2. recognize static control parts;

- affine loop bounds, conditionals and array subscripts;

- build polyhedral domains, sequential schedules and array accesses;

- graceful degradation when all conditions are not met;

3. apply WRaP analyses and transformations;

4. polyhedral code generation, modifying CLooG to output WHIRL;

- generate new loops, conditionals and variables;

- move/duplicate the original statement nodes;

- optimize conditionals;

5. resume the compilation flow, redoing scalar optimizations, or regenerate source code with Open64 tools (whirl2c or whirl2f).

Although WRaP-IT is still a prototype, it proved to be very robust and the whole source-to-polyhedra-to-source transformation was successfully applied to all 12 benchmarks in Figure 2. All the tools are free software, and further documentation and information can be found on http://www-rocq.inria.fr/a3/wrap-it.

\section{Conclusion}

We described a theoretical framework to streamline the design of polyhedral transformations, based on a unified polyhedral representation and a set of transformation

$\mathrm{RR} \mathrm{n}^{\circ} 4902$ 
primitives. It decouples the transformation from the static analyses. It is intended as a formal tool for semi-automatic optimization, where program transformations with the associated static analyses for semantic-preservation - are separated from the optimization or parallelization algorithm which drives the transformations and select their parameters.

We also described WRaP-IT, a robust tool to convert back and forth between Fortran or $\mathrm{C}$ source and the polyhedral representation. This tool is implemented in Open64/ORC. The complexity of the code generation phase, when converting back to source code, has long been a deterrent for using polyhedral representations in optimizing or parallelizing compilers. However, our code generator (CLooG) can handle loops with more than 1700 statements. Moreover, the whole source-to-polyhedrato-source transformation was successfully applied to the 12 benchmarks. This is a strong point in favor of polyhedral techniques, even in the context of real codes.

Current and future work include the design and implementation of a polyhedral transformation library, an iterative compilation scheme with a machine-learning algorithm and/or an empirical optimization methodology, and the optimization of the code generator to keep producing optimal code on larger codes.

\section{References}

[1] T. Kisuki, P. Knijnenburg, M. O'Boyle, and H. Wijshoff. Iterative compilation in program optimization. In Proc. CPC'10 (Compilers for Parallel Computers), pages $35-44,2000$.

[2] M. O'Boyle, P. Knijnenburg, and G. Fursin. Feedback assisted iterative compiplation. In Parallel Architectures and Compilation Techniques (PACT'01). IEEE Computer Society Press, October 2001.

[3] K. Yotov, X. Li, G. Ren, M. Cibulskis, G. DeJong, M. Garzaran, D. Padua, K. Pingali, P. Stodghill, and P. Wu. A comparison of empirical and modeldriven optimization. In ACM Symp. on Programming Language Design and Implementation (PLDI'03), San Diego, California, USA, June 2003.

[4] Open research compiler. http://ipf-orc.sourceforge.net.

[5] C. Bastoul and P. Feautrier. Improving data locality by chunking. In $C C^{\prime} 12$ International Conference on Compiler Construction, LNCS 2622, pages 320335, Warsaw, Poland, april 2003. 
[6] Keith D. Cooper, Mary W. Hall, Robert T. Hood, Ken Kennedy, Kathryn S. McKinley, John M. Mellor-Crummey, Linda Torczon, and Scott K. Warren. The ParaScope parallel programming environment. Proceedings of the IEEE, 81(2):244-263, 1993.

[7] M. Hall et al. Maximizing multiprocessor performance with the SUIF compiler. IEEE Computer, 29(12):84-89, December 1996.

[8] W. Blume, R. Eigenmann, K. Faigin, J. Grout, J. Hoeflinger, D. Padua, P. Petersen, W. Pottenger, L. Rauchwerger, P. Tu, and S. Weatherford. Parallel programming with Polaris. IEEE Computer, 29(12):78-82, December 1996.

[9] F. Irigoin, P. Jouvelot, and R. Triolet. Semantical interprocedural parallelization: An overview of the pips project. In ACM Int. Conf. on Supercomputing (ICS'2), Cologne, Germany, June 1991.

[10] W. Kelly, W. Pugh, and E. Rosser. Code generation for multiple mappings. In Frontiers'95 Symposium on the frontiers of massively parallel computation, McLean, 1995.

[11] W. Kelly. Optimization within a unified transformation framework. Technical Report CS-TR-3725, University of Maryland, 1996.

[12] A.-C. Guillou, F. Quilleré, P. Quinton, S. Rajopadhye, and T. Risset. Hardware design methodology with the alpha language. In $F D L^{\prime} 01$, Lyon, France, September 2001.

[13] R. Schreiber, S. Aditya, B. Rau, V. Kathail, S. Mahlke, S. Abraham, and G. Snider. High-level synthesis of nonprogrammable hardware accelerators. Technical report, Hewlett-Packard, May 2000.

[14] P. Feautrier. Dataflow analysis of scalar and array references. Int. Journal of Parallel Programming, 20(1):23-53, February 1991.

[15] J.-F. Collard, D. Barthou, and P. Feautrier. Fuzzy array dataflow analysis. In ACM Symp. on Principles and Practice of Parallel Programming, pages 92-102, Santa Barbara, California, USA, July 1995.

[16] D. Wonnacott and W. Pugh. Nonlinear array dependence analysis. In Proc. Third Workshop on Languages, Compilers and Run-Time Systems for Scalable Computers, 1995. Troy, New York, USA.

$\operatorname{RR} \mathrm{n}^{\circ} 4902$ 
[17] L. Rauchwerger and D. Padua. The lrpd test: Speculative run-time parallelization of loops with privatization and reduction parallelization. IEEE Transactions on Parallel and Distributed Systems, Special Issue on Compilers and Languages for Parallel and Distributed Computers, 10(2):160-180, 1999.

[18] R. Eigenmann, J. Hoeflinger, and D. Padua. On the automatic parallelization of the perfect benchmarks. IEEE Trans. on Parallel and Distributed Systems, 9(1):5-23, January 1998.

[19] A. Schrijver. Theory of Linear and Integer Programming. John Wiley and Sons, Chichester, UK, 1986.

[20] B. Creusillet and F. Irigoin. Interprocedural array region analyses. Int. Journal of Parallel Programming, 24(6):513-546, December 1996.

[21] P. Feautrier. Some efficient solution to the affine scheduling problem, part II, multidimensional time. Int. Journal of Parallel Programming, 21(6):389-420, December 1992. See also Part I, One Dimensional Time, 21(5):315-348.

[22] C. Ancourt and F. Irigoin. Scanning polyhedra with DO loops. In 3rd ACM SIGPLAN Symposium on Principles and Practice of Parallel Programming, pages 39-50, june 1991.

[23] W. Li and K. Pingali. A singular loop transformation framework based on nonsingular matrices. International Journal of Parallel Programming, 22(2):183205, April 1994.

[24] J. Xue. Automating non-unimodular loop transformations for massive parallelism. Parallel Computing, 20(5):711-728, 1994.

[25] M. Griebl, C. Lengauer, and S. Wetzel. Code generation in the polytope model. In PACT'98 International Conference on Parallel Architectures and Compilation Techniques, pages 106-111, 1998.

[26] P. Boulet, A. Darte, G-A. Silber, and F. Vivien. Loop parallelization algorithms: From parallelism extraction to code generation. Parallel Computing, 24(3):421444, 1998.

[27] F. Quilleré, S. Rajopadhye, and D. Wilde. Generation of efficient nested loops from polyhedra. International Journal of Parallel Programming, 28(5):469-498, october 2000 . 


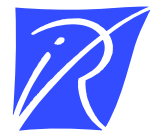

Unité de recherche INRIA Rocquencourt

Domaine de Voluceau - Rocquencourt - BP 105 - 78153 Le Chesnay Cedex (France)

Unité de recherche INRIA Futurs : Domaine de Voluceau - Rocquencourt - BP 105 - 78153 Le Chesnay Cedex (France) Unité de recherche INRIA Lorraine : LORIA, Technopôle de Nancy-Brabois - Campus scientifique 615, rue du Jardin Botanique - BP 101 - 54602 Villers-lès-Nancy Cedex (France)

Unité de recherche INRIA Rennes : IRISA, Campus universitaire de Beaulieu - 35042 Rennes Cedex (France)

Unité de recherche INRIA Rhône-Alpes : 655, avenue de l'Europe - 38330 Montbonnot-St-Martin (France)

Unité de recherche INRIA Sophia Antipolis : 2004, route des Lucioles - BP 93 - 06902 Sophia Antipolis Cedex (France)

INRIA - Domaine de Voluceau - Rocquencourt, BP 105 - 78153 Le Chesnay Cedex (France)

http://www.inria.fr

ISSN 0249-6399 